\title{
Validez discriminante, predictiva e incremental de la escala de comportamientos laborales proactivos de Belschak y Den Hartog* Discriminant, Predictive and Incremental Validity of Belschak \& Den Hartog's Proactive Work Behaviors Scale
}

\author{
Solana Salessi, \\ Alicia Omar \\ Consejo Nacional de Investigaciones Científicas y \\ Técnicas, Argentina
}

\begin{abstract}
Resumen
El objetivo de este estudio fue establecer la validez discriminante, predictiva e incremental de la Escala de Comportamientos Laborales Proactivos de Belschak y Den Hartog. Se realizó un estudio empírico-instrumental con una muestra no probabilística (510 trabajadores, 53\% varones). Análisis de ecuaciones estructurales exploratorios (ESEM) mostraron saturaciones cruzadas inferiores a $.30 \mathrm{y}$ un adecuado ajuste de un modelo hexafactorial oblicuo. Los valores de varianza media extraída, de su raíz cuadrada y de la proporción heterorrasgo-monorrasgo proporcionaron evidencia de validez discriminante. Análisis de regresión con SEM confirmaron la contribución significativa de los comportamientos proactivos en la explicación de la satisfacción y de la implicación en el trabajo. Del mismo modo, la personalidad proactiva mostró ser un antecedente significativo de los comportamientos proactivos. Los resultados brindan evidencia favorable a la validez del instrumento analizado.
\end{abstract}

Palabras clave: proactividad, satisfacción laboral, implicación en el trabajo, validez, estudio instrumental

*Este trabajo ha sido realizado en el marco de la beca posdoctoral otorgada por el Consejo Nacional de Investigaciones Científicas y Técnicas (CONICET) a la primera autora bajo la dirección de la segunda.

Solana Salessi, Consejo Nacional de Investigaciones Científicas y Técnicas (CONICET), Instituto de Investigaciones de la Facultad de Humanidades y Artes de la Universidad Nacional de Rosario, Argentina; Alicia Omar, Consejo Nacional de Investigaciones Científicas y Técnicas (CONICET), Instituto de Investigaciones de la Facultad de Humanidades y Artes de la Universidad Nacional de Rosario, Argentina.

La correspondencia en relación con este artículo se dirige a Solana Salessi, Facultad de Derecho y Ciencias Sociales del Rosario, Pontificia Universidad Católica Argentina. Dirección electrónica: solanasalessi@uca.edu.ar 


\begin{abstract}
:
The aim of this study was to establish the discriminant, predictive and incremental validity of Belschak \& Den Hartog's Proactive Work Behaviors Scale. An empirical-instrumental study was carried out with a non-probabilistic sample (510 workers, 53\% males). Analysis of exploratory structural equations (ESEM) showed cross-saturations lower than .30 and an adequate adjustment of an oblique hexa-factorial model. The values of average variance extracted, of its square root and of the heterotrait-monotrait ratio provided evidence of discriminant validity. SEM regression analysis confirmed the significant contribution of proactive behaviors in the explanation of job satisfaction and job involvement. Proactive personality showed itself to be a significant predictor of proactive behaviors. These results obtained provide favorable evidence for the validity of the instrument analyzed.
\end{abstract}

Keywords: Proactivity, Job Satisfaction, Job Involvement, Validity, Instrumental Study.

En los últimos años, el análisis de la validez se ha posicionado como un tópico crucial en la investigación psicológica en general y organizacional en particular. De acuerdo con Henseler, Ringle y Sarstedt (2015) este renovado interés posiblemente se deba a la popularidad que han adquirido en estas disciplinas los métodos basados en la estructura de varianzas y covarianzas tales como el enfoque SEM (del inglés Structural Equation Modeling). Estos métodos utilizan compuestos lineales de los ítems como indicadores observables de los factores latentes; es decir, el puntaje en el instrumento es una medida compuesta que se genera a partir de las puntuaciones individuales para cada ítem y que indica el nivel que toma el constructo en los sujetos examinados. Para que la variabilidad entre los puntajes se deba a diferencias reales en el constructo y no a sesgos metodológicos, es indispensable que los modelos de medición utilizados por los investigadores representen a los constructos que pretenden medir de una manera válida (Montero-Rojas, 2013).

El término "validez" representa un concepto complejo y controvertido que ha sido discutido tanto en términos pragmáticos como éticos-filosóficos. Entre los exponentes del enfoque pragmático se encuentran los autores calificados de "ultraconservadores" (Newton \& Shaw, 2016). Vale decir, aquellos que sostienen que la validez es una propiedad de los test y no de las interpretaciones, los puntajes o los constructos (Borsboom, Mellenbergh, \& Van Heerden, 2004). Entre los autores enmarcados en un posicionamiento ético-filosófico se ubican aquellos que Newton y Shaw (2016) denominan "liberales". Se trata de quienes conciben a la validez como el grado en el cual la evidencia empírica y la teoría sustentan que las inferencias y las acciones basadas en los puntajes de una prueba son adecuados y apropiados (Messick, 1980).

Los requerimientos de validez atraviesan todas las áreas de la investigación psicológica, por lo que los estudios sobre comportamiento organizacional no se encuentran exentos de tales exigencias. Numerosos especialistas (Le, Schmidt, Harter, \& Lauver, 2010; Morrow, 1983; Rousseau, 2007; Schwab, 1980) han subrayado la necesidad de analizar la redundancia entre constructos, con miras a evitar la proliferación indiscriminada de conceptos y garantizar la parsimonia. Mención especial, en este sentido, merece el término proactividad. A este respecto diversos autores (Thomas, Whitman, \& Viswesvaran, 2010; Tornau \& Frese, 2013) advierten sobre la posible superposición entre los constructos que integran su red nomológica. Tal es el caso de los conceptos de personalidad proactiva (Bateman \& Crant, 1993) y comportamientos proactivos (Belschak \& Den Hartog, 2010; Parker \& Collins, 2010). 
Escala Comportamientos Proactivos: evidencias de validez

Si bien distintos estudios han informado evidencias de validez (Bateman \& Cran, 1993; Belschak \& Den Hartog, 2010; 2017; Crant, Hu \& Jiang, 2017; Parker \& Collins, 2010), hasta el momento, no se registran investigaciones centradas en analizarlos conjuntamente, así como en determinar el aporte específico de cada medida en relación con resultados organizacionalmente deseables; por ejemplo, la satisfacción laboral y la implicación en el trabajo. En este sentido, las investigaciones sobre la influencia de la personalidad proactiva y de los comportamientos proactivos han discurrido por carriles paralelos, aisladas unas de otras. Como corolario, exiguos estudios se han abocado a determinar el aporte e importancia relativa de cada medida. Establecen en qué grado su inclusión proporciona una mejora sustancial en el modelo (Hunsley \& Meyer, 2003; Sackett, Dahlke, Shewach, \& Kuncel, 2017). Frente a este vacío empírico, la presente investigación se propuso explorar la validez discriminante, predictiva e incremental de la adaptación argentina (Salessi \& Omar, 2018a) de la Escala de Comportamientos Laborales Proactivos de Belschak y Den Hartog (2010).

De acuerdo con los estándares para el uso de pruebas a los que adscriben organismos referentes en educación y psicología (American Psychological Association, American Educational Research Association, \& National Council on Measurement in Education, 2014), todo estudio instrumental, como el presente, debe recolectar evidencias de diversas fuentes. Entre estas, la evidencia basada en la estructura interna de la prueba y la evidencia basada en criterios externos constituyen el eje de este trabajo. La primera responde al análisis estadístico de los ítems para examinar, por ejemplo, las dimensiones medidas en una evaluación, la consistencia interna de la prueba, etc. La segunda, en tanto, se basa en las relaciones con otras variables. Se contemplan las formas tradicionales de validez, usualmente catalogadas como validez concurrente y predictiva. Con miras a proporcionar este segundo tipo de evidencias, la presente investigación ha considerado medidas estandarizadas de personalidad proactiva (Bateman \& Crant, 1993; Salessi \& Omar, en prensa), satisfacción laboral (Salessi \& Omar, 2016) e implicación en el trabajo (Salessi \& Omar, 2018b).

\section{Personalidad proactiva y comportamientos proactivos como constructos distinguibles}

Para ser considerados diferentes dos constructos deben ser distinguibles tanto conceptual como empíricamente. En lo que hace al primer requisito, se ha objetado (Le, Schmidt, Harter, \& Lauver, 2010) que es algo débil y, en general, puede satisfacerse fácilmente. En efecto, la suposición que subyace a este criterio es que, si los investigadores pueden hacer una distinción lógica entre los constructos, esta distinción también existirá en la "mente" de los participantes del estudio. Sin embargo, esta aseveración puede no ser válida (Harter \& Schmidt, 2008). El segundo requerimiento, en tanto, sostiene que dos constructos supuestamente distintos no deben correlacionar entre sí de modo "perfecto", una vez que los efectos producidos por los sesgos de la medición han sido controlados. Si las correlaciones entre dos constructos son próximas a 1, tales constructos son indiferenciables; vale decir, empíricamente redundantes (Le et al., 2010; Morrow, 1983; Rousseau, 2007; Schwab, 1980).

En relación con los conceptos bajo análisis, es posible plantear algunas diferencias que sustenten teóricamente la validez discriminante. La más importante se centra en que, mientras el concepto de personalidad proactiva se inscribe en una perspectiva de rasgo (Bateman \& Crant, 1993; Crant et al., 2017), la noción de comportamientos proactivos se encuadra en un enfoque situacional (Parker \& Bindl, 2017; 
Parker \& Collins, 2010; Torneau \& Frese, 2013). En este sentido, en su artículo seminal Bateman y Crant definen explícitamente a la personalidad proactiva como "un componente disposicional" (Crant et al., 2017, p. 198). De este modo, el concepto de personalidad proactiva acentúa las diferencias interpersonales en la predisposición a influir sobre el entorno y se constituye en una tendencia relativamente estable a generar cambios en el ambiente, independientemente de las características de estos (Salessi \& Omar, en prensa).

Más allá de los hallazgos preliminares comunicados por Bateman y Crant (1993) sobre la estabilidad de la proactividad en el tiempo, pocos estudios se han expedido sobre esta cuestión. Alguno recientes provienen del campo de la genética conductual y proveen cierta evidencia consistente con esta conceptualización. Por ejemplo, algunas investigaciones (Liu, 2013, 2015, citados en Crant et al., 2017) han informado que el $42 \%$ de la varianza de la personalidad proactiva es atribuible a diferencias genéticas, y que cierto marcador genético (DAT1) está asociado a la personalidad proactiva. Tales hallazgos, aunque exiguos, resultan alentadores para quienes defienden la naturaleza disposicional de la proactividad.

Sin embargo, diversos especialistas (Belschak \& Den Hartog, 2017; Grant \& Ashford, 2008; Parker \& Bindl, 2017; Parker \& Collins, 2010) advierten que esta concepción se focaliza en la personalidad de los individuos que tienden a comportarse proactivamente, en lugar de centrarse en las conductas en sí mismas. De este modo, ofrece muy poca información respecto a qué comportamientos deberían ser clasificados como proactivos. Así, podría presuponerse que los comportamientos proactivos son, exclusivamente, conductas extrarroles cuando, en realidad, todos los tipos de desempeño (sean ejecuciones inherentes al rol o acciones que trascienden sus requerimientos formales) pueden ser más o menos proactivos (Belschak \& Den Hartog, 2017; Grant \& Ashford, 2008).

Los representantes del enfoque situacional consideran que los comportamientos proactivos constituyen "una forma de comportarse que puede variar de acuerdo con las circunstancias" (Parker \& Wang, 2015, p. 63). En efecto, las notas distintivas de la proactividad pueden reducirse, esencialmente, a tres aspectos: (a) que se trate de comportamientos impulsados por motivaciones intrínsecas antes conductas coaccionadas; (b) que tales comportamientos se encuentren centrados en el futuro, es decir, relacionados a la anticipación de oportunidades y a la prevención de problemas y (c) que tales acciones estén orientadas a producir cambios en uno mismo, en el entorno o en los demás (Parker \& Bindl, 2017). En función de los objetivos perseguidos (Belschak \& Den Hartog, 2010; 2017), pueden distinguirse: (a) comportamientos proindividuales u orientados a uno mismo (centrados en mejorar el propio desempeño del empleado); (b) comportamientos prosociales u orientados a los demás (direccionados a potenciar el rendimiento de los compañeros $\mathrm{y}$, en última instancia, del equipo) y (c) comportamientos proorganizacional u orientados a la organización (focalizados en contribuir al logro de la misión y de la visión organizacional). Con base en estos antecedentes, se hipotetiza que:

Hipótesis 1: los comportamientos proactivos (tal como son operacionalizados por la Escala de Belschak y Den Hartog, 2010) serán empíricamente distinguibles de la personalidad proactiva (tal como es operacionalizada por la Escala de Bateman y Crant, 1993). 
Escala Comportamientos Proactivos: evidencias de validez

\section{Personalidad y comportamientos proactivos como predictores de actitudes y conductas}

En lo que respecta a la validez predictiva de la proactividad, la literatura especializada indica que se asocia a un amplio espectro de resultados favorables vinculados tanto al desempeño individual de los trabajadores como al éxito de las organizaciones. En relación con la personalidad proactiva, evidencias exponen que se vincula a mayor prosperidad en el trabajo y adaptabilidad profesional (Jiang, 2017). Del mismo modo, numerosos estudios (Liao, 2015; Vandenberghe \& Ok, 2013; Wang, Zhang, Thomas, Yu, \& Spitzmueller, 2017; Yang, Yan, Fan, \& Luo, 2017) señalan que se relaciona positivamente con la integración social, las intenciones de permanecer en la empresa, el compromiso con la organización y el engagement. De forma paralela, recientes investigaciones (Salessi \& Omar, en prensa; Turban, Moake, $\mathrm{Wu}$, Cheung, \& Yu, 2017) informan mayor satisfacción profesional e involucramiento personal entre trabajadores con personalidad proactiva. Finalmente, distintos autores (Crant et al., 2017; Bergeron, Schroeder, \& Martinez, 2014; Parker \& Collins, 2010; Spitzmuller, Sin, Howe \& Fatimah, 2015) subrayan que constituye un antecedente preponderante de numerosos comportamientos proactivos específicos, tales como los comportamientos de ciudadanía organizacional, la innovación, la prevención de problemas y la asunción de control. Frente a estos hallazgos se conjetura que:

Hipótesis 2: la personalidad proactiva explicará una varianza significativa de la satisfacción laboral, la implicación en el trabajo, y los comportamientos proactivos proindividuales, prosociales y proorganizacionales.

En lo que hace a las conductas proactivas, la evidencia científica indica que también tienen repercusiones favorables para el comportamiento individual y para la competitividad de las organizaciones. En este sentido, Cooper-Thomas, Paterson, Stadler y Saks (2014) han demostrado que los comportamientos proactivos se asocian a mayores niveles de bienestar, apertura al aprendizaje y engagement. Por su parte, Cangiano y Parker (2016) han destacado que, en presencia de elevados niveles de motivación intrínseca y retroalimentación positiva por parte del entorno, comportarse proactivamente promueve mayor autoeficacia, autodeterminación y emociones positivas. En sintonía con estos resultados, algunos autores (Ismaeli, Nowalid, \& Bakar, 2016; Mallin, Ragland, \& Finkle, 2014; Salessi \& Omar, 2018a) han encontrado evidencia preliminar sobre las vinculaciones positivas entre los comportamientos proactivos, la implicación con el trabajo y la satisfacción laboral.

Sumado a lo anterior, algunas investigaciones manifiestan la validez incremental de los comportamientos proactivos en la explicación de variables organizacionales clave. En este sentido, por ejemplo, Torneau y Frese (2013) han encontrado que la asunción de control, la iniciativa personal y las conductas de voz superan ampliamente la contribución atribuible a la personalidad proactiva en la predicción del desempeño. En la misma línea, Belschak y Den Hartog (2010) han comunicado que los comportamientos proactivos pro individuales y proorganizacionales realizan un aporte predictivo incremental a la explicación del rendimiento. A partir de estos referentes empíricos se presupone que:

Hipótesis 3: los comportamientos proactivos proindividuales, prosociales y proorganizacionales realizarán un aporte significativo positivo e incremental (más allá de la personalidad proactiva) a la predicción de la satisfacción laboral y de la implicación en el trabajo. 


\section{Método}

\section{Diseño}

Se realizó un estudio de corte transversal, descriptivo-correlacional, encuadrado en la categoría de estudios instrumentales (Ato, López \& Benavente, 2013).

\section{Participantes}

Se integró una muestra no probabilística (por disponibilidad) de 556 participantes. Treinta y dos casos fueron descartados por presentar protocolos incompletos. La muestra definitiva quedó, entonces, conformada por 524 trabajadores (53\% varones) de diversas organizaciones argentinas públicas y privadas localizadas en el centro-sur de la provincia de Santa Fe y norte de la provincia de Buenos Aires. El promedio de edad fue 34 años $(D T=12.74)$. La antigüedad laboral media fue 6 años $(D T=9.21)$. El $74.5 \%$ de la muestra tenía estudios superiores terciarios y/ o universitarios. El 56\% trabajaba en el sector privado. La distribución por rubros de actividad fue la siguiente: $34.2 \%$ comercio; $28.5 \%$ industria; $22.6 \%$ salud y $14.7 \%$ educación.

\section{Procedimiento}

La recolección de los datos se llevó a cabo en el seno de las organizaciones que aceptaron participar de la investigación, en días y horarios previamente pautados con las autoridades y en los lugares físicos que las mismas dispusieron a tal efecto. La participación de los trabajadores fue voluntaria, anónima y confidencial. No se brindaron incentivos de ningún tipo. La administración de las técnicas estuvo a cargo de personal entrenado a tal efecto. En todos los casos, los participantes completaron de manera individual un cuadernillo conteniendo en su primera hoja el objetivo del estudio y las instrucciones para responder; en la segunda hoja, el formulario de consentimiento informado y en las restantes páginas, los ítems correspondientes a los instrumentos que a continuación se describen distribuidos aleatoriamente. La ejecución del estudio se desarrolló en un todo de acuerdo con los principios éticos establecidos por el Código de Conducta de la Asociación Americana de Psicología (2017) y con las recomendaciones del Consejo Nacional de Investigaciones Científicas y Técnicas para las investigaciones en las ciencias sociales y humanas (CONICET, 2006, Resolución N. ${ }^{\circ} 2857$ ).

\section{Instrumentos}

Adaptación argentina de la Escala de Comportamientos Laborales Proactivos (Salessi \& Omar, 2018a) de Belschaky Den Hartog (2010). El instrumento evalúa los comportamientos proactivos, que se entienden como conductas automotivadas orientadas al cambio constructivo, que pretenden influir sobre la organización, los demás o uno mismo. La escala está integrada por 10 ítems presentados en formato Likert de 5 puntos $(1=$ totalmente en desacuerdo; $5=$ totalmente de acuerdo $)$. Los reactivos se distribuyen a lo largo de tres subescalas denominadas: comportamientos proactivos proorganizacionales (3 ítems; ej.: "sugiero ideas frente a los problemas que afronta mi organización"; $\alpha=.80$ ); comportamientos proactivos prosociales (4 ítems; ej.: "ayudo a mis compañeros para que desarrollen e implementen nuevas ideas"; $\alpha=.82$ ) y comportamientos proactivos proindividuales ( 3 ítems; ej.: "encuentro nuevas maneras de realizar mis tareas y ser más efectivo" $\alpha=.81$ ). 
Escala Comportamientos Proactivos: evidencias de validez

Adaptación argentina de la Escala de Personalidad Proactiva (Salessi \& Omar, en prensa) de Bateman y Crant (1993). El instrumento evalúa la personalidad proactiva, definida como una tendencia estable a generar cambios en el ambiente. La versión adaptada está integrada por 6 ítems (ej.: "siempre estoy buscando formas de hacer mejor las cosas"; $=\alpha=.82$ ) valorados sobre una escala tipo Likert de 5 puntos $(1=$ totalmente en desacuerdo; 5 = totalmente de acuerdo $)$.

Adaptación argentina de la Escala de Satisfacción Laboral Genérica (Salessi \& Omar, 2016) de Mac Donald y Mac Intyre (1997). El instrumento evalúa la satisfacción laboral entendida como una actitud general; vale decir, un conjunto de respuestas cognitivas y afectivas frente la situación laboral. La escala está integrada por 7 ítems (ej.: "en mi trabajo puedo aplicar todas mis capacidades y habilidades" $\alpha=.87$ ) valorados sobre una escala tipo Likert de 5 puntos $(1=$ totalmente en desacuerdo; $5=$ totalmente de acuerdo $)$.

Adaptación argentina del Cuestionario de Implicación en el Trabajo (Salessi \& Omar, 2018b) de Kanungo (1992). El instrumento evalúa la implicación laboral definida como el grado en que una persona está cognitiva y emocionalmente comprometida e involucrada con su trabajo actual. La escala está integrada por 9 ítems (ej.: "mi trabajo ocupa un lugar irremplazable en mi vida"; $\alpha=.82$ ) valorados sobre una escala tipo Likert de 5 puntos $(1=$ totalmente en desacuerdo; $5=$ totalmente de acuerdo $)$.

\section{Estrategia de análisis de datos}

Para el procesamiento de los datos se utilizaron los programas SPSS (versión 23.0), Mplus (versión 7.3) y G*Power. La estrategia implementada para el análisis se desarrolla a continuación.

\section{Análisis preliminares}

Se examinó la matriz de datos con miras a detectar valores perdidos y/o extremos. Se identificaron los casos atípicos mediante el cálculo de puntuaciones $Z$ y las distancias de Mahalanobis al cuadrado $\left(D^{2}\right)$ para cada variable. Se consideraron atípicas univariadas aquellas observaciones alejadas más de 3.5 DT de la media y atípicas multivariadas a aquellas que presentasen $D^{2}$ con probabilidad igual o inferior a .001 (Hair, Black, Babin, Anderson, \& Tatham, 2010; Tabachnick \& Fidell, 2013). Se realizó un análisis de los ítems mediante el cálculo de estadísticos descriptivos (medias y desviaciones típicas), coeficientes de asimetría y curtosis e índices de discriminación (a partir del cómputo de las correlaciones ítem-total corregidas). Valores de asimetría y curtosis inferiores a \pm 2.00 y correlaciones positivas superiores a .30 se consideraron adecuadas (Tabachnick \& Fidell, 2013). Por último, se examinó el cumplimiento del supuesto de independencia entre los residuos (mediante el estadístico $d$ de Durbin-Watson); de los supuestos de homocedasticidad, linealidad, normalidad (a través de la inspección de los diagramas de dispersión de los pronósticos tipificados, de los residuos tipificados, del histograma de los residuos tipificados, del gráfico de probabilidad normal y del diagrama de regresión parcial) y del supuesto de libre colinealidad (mediante el índice de tolerancia y su inverso FIV). Se consideraron óptimos valores del estadístico $d$ comprendidos entre 1.5 y 2.5 ; valores superiores a .10 en el índice de tolerancia y valores inferiores a 3 en su inverso FIV (Tabachnick \& Fidell, 2013; Hair, Black, Babin, Anderson, \& Tatham, 2010).

\section{Análisis de ecuaciones estructurales exploratorio}

Con miras a determinar si los ítems son indicadores operacionales adecuados de los constructos que pretenden medir, se realizó un análisis de ecuaciones estructurales exploratorio (ESEM, del inglés 
Exploratory Structural Equation Modelling). Este método genera estimaciones más precisas de las cargas factoriales y las correlaciones entre factores al permitir la estimación libre de las cargas cruzadas. Por tanto, es menos restrictivo que el análisis factorial confirmatorio tradicional y se ajusta más a la realidad de las medidas psicológicas, donde habitualmente los ítems no constituyen indicadores "perfectos" del constructo y presentan saturaciones menores (aunque no nulas) en los otros factores (Marsh, Morin, Parker, \& Kaur, 2014).

Para la estimación del modelo se empleó el método de Mínimos Cuadrados no Ponderados con Ajuste en Medias y Varianzas (WLSMV, del inglés Weighted Least Squares Means and Variance Adjusted). Asimismo, se utilizó rotación oblicua Target, porque proporciona la solución rotada más próxima a una configuración preespecificada de cargas. Así, permite utilizar el ESEM en un modo confirmatorio. Para evaluar la bondad de ajuste, se analizó que: (a) el Índice de Bondad de Ajuste (GFI, Goodness of Fit Índex), el Índice deAjuste Comparativo(CFI, Comparative Fit Índex) y elIIndicede Tucker-Lewis(TLI, Tucker-Lewis Índex) alcanzaran valores iguales o superiores a .90y (b) que el Error Cuadrático Medio deAproximación(RMSEA, Root Mean Square Error of Approximation) fuera inferior a .08 (Hair et al., 2010; Marsch et al., 2014).

\section{Validez discriminante}

Se obtuvo evidencia de validez discriminante mediante tres procedimientos: (a) el cálculo de la Varianza Media Extraída (AVE, del inglés Average Variance Extracted) y de su raíz cuadrada, respectivamente; (b) el cómputo de la Proporción Heterorasgo-Monorasgo (HTMT, del inglés HeterotraitMonotrait Ratio) y (c) el cálculo de las correlaciones entre los constructos estudiados. El AVE posibilita estimar la varianza común entre los indicadores y su factor latente. Se consideran apropiados valores iguales o superiores a .50. Asimismo, valores de su raíz cuadrada superiores a la correlación entre los factores latentes, se asumen como evidencia de que cada constructo comparte más varianza con sus indicadores que con los demás (Cheung \& Chang, 2017; Henseler et al., 2015; Martínez-García \& MartínezCaro, 2009). La proporción HTMT, en tanto, es la media de las correlaciones entre los indicadores que miden diferentes constructos (correlación HT, del inglés Heterotrait-Heteromethod), en relación con el promedio de las correlaciones de indicadores dentro del mismo constructo (correlaciones MT, del inglés Monotrait-Heteromethod). Los valores inferiores a .90 demuestran una adecuada validez discriminante (Henseler et al., 2015).

\section{Validez, predictiva e incremental}

Para analizar la contribución predictiva e incremental de los comportamientos proactivos en la explicación de la satisfacción laboral y de la implicación en el trabajo, se estimó el ajuste de dos modelos mediante análisis de ecuaciones estructurales. El primer modelo incluyó como variable exógena a la personalidad proactiva. En el segundo modelo se añadieron, además, los comportamientos proactivos proindividuales, prosociales y pro organizacionales, respectivamente. Asimismo, con el fin de analizar si la personalidad proactiva explica una porción significativa de la varianza de los comportamientos proactivos, se estimó un tercer modelo. En esta oportunidad, los tres tipos de comportamientos proactivos se modelaron como variables endógenas. En todos los casos, las características sociodemográficas (edad, antigüedad laboral, sexo, nivel académico, ramo de actividad y sector organizacional) fueron consideradas variables control. Todas las variables nominales fueron recategorizadas como variables 
Escala Comportamientos Proactivos: evidencias de validez

dummy (sexo: 1 = varón, 2 = mujer; nivel académico; 1 = sin estudios superiores, $2=$ con estudios superiores; ramo de actividad: 1 = comercio/servicios, 2 = industria y sector organizacional: $1=$ público, 2 = privado).

Los tres modelos fueron estimados mediante el método WLSMV. La bondad de ajuste se evaluó mediante los índices CFI, TLI y RMSEA. Paralelamente, se analizaron el $R^{2}$ y el tamaño del efecto; este último computa el coeficiente $f^{2}$ de Cohen. La magnitud del efecto se determinó al interpretar que valores de $02, .15$ y .35 se consideran pequeños, medianos y grandes, respectivamente (Cohen, 1992).

\section{Resultados}

\section{Análisis exploratorios}

El porcentaje de datos perdidos en cada ítem no superó el 5\%. Los valores faltantes fueron tratados mediante el método de Estimación-Maximización (Tabachnick \& Fidell, 2013). Se detectaron 14 casos atípicos, 9 de los cuales satisfacían la condición para considerarse atípicos multivariados (Hair et al., 2010; Tabachnick \& Fidell, 2013). Por lo tanto, fueron eliminados y quedó la muestra final constituida por 510 participantes, que se trata de un tamaño adecuado para la ejecución de los análisis previstos. Los gráficos indicaron el cumplimiento de los supuestos de homocedasticidad, linealidad y normalidad. Se obtuvieron valores de tolerancia entre .32 y .64, y entre 1.20 y 1.65 en su inverso FIV. El estadístico $d$ fue de 1.24. Tales resultados mostraron un adecuado cumplimiento de los supuestos multivariados (Hair et al., 2010; Tabachnick \& Fidell, 2013). No se registraron problemas de asimetría y curtosis. Además, las correlaciones ítem-total fueron todas positivas. Los valores correspondientes se informan en la Tabla 1.

\section{Análisis de ecuaciones estructurales exploratorio}

Los índices obtenidos $(\mathrm{GFI}=.90, \mathrm{CFI}=.91, \mathrm{TLI}=.91, \mathrm{RMSEA}=.04, \mathrm{IC} 95 \%[.03, .05])$ para el modelo de medida hexa-factorial (seis factores correlacionados: personalidad proactiva, comportamientos proactivos proindividuales, comportamientos proactivos prosociales, comportamientos proactivos proorganizacionales, implicación en el trabajo y satisfacción laboral) indicaron un adecuado ajuste (Marsch et al., 2014; Hair et al., 2010). Las saturaciones de los ítems en cada factor se presentan en la tabla 1. 
Escala Comportamientos Proactivos: evidencias de validez

Como puede observarse, en todos los casos, los ítems pesaron más en el factor latente que corresponde. Un análisis más pormenorizado muestra que el ítem 5 ("siempre estoy buscando formas de hacer mejor las cosas") perteneciente al factor personalidad proactiva presentó las saturaciones cruzadas más elevadas en el factor 4 (comportamientos proactivos proindividuales). Del mismo modo, el ítem 14 ("busco nuevos modos de realizar mi trabajo para ser más eficiente"), que compete al factor comportamientos proactivos proindividuales, mostró saturaciones cruzadas más altas en el factor personalidad proactiva. Sin embargo, en ninguno de los dos casos las cargas fueron superiores a .30 (Marsch et al., 2014). Ello proporciona evidencia preliminar favorable a la validez discriminante entre las escalas analizadas.

\section{Análisis de validez discriminante}

La tabla 2 muestra los coeficientes de correlación, los valores correspondientes al índice AVE, a su raíz cuadrada y a la proporción HTMT.

De los resultados se desprende que las puntuaciones en la escala de comportamientos proactivos mostraron relaciones positivas con las puntuaciones en las escalas de personalidad proactiva, implicación en el trabajo y satisfacción laboral. No obstante, para los comportamientos proindividuales, las correlaciones fueron ligeramente superiores. Las raíces cuadradas del AVE correspondientes a cada variable latente de comportamiento proactivo fueron mayores a la correlación entre estos y los restantes

Tabla 2

Estadísticos descriptivos, índices de correlación, varianza media extraída y proporción heterorrasgo-monorrasgo de las variables bajo estudio

\begin{tabular}{lcccccccccc}
\hline & $M$ & $D T$ & 1 & 2 & 3 & 4 & 5 & 6 & AVE & $\sqrt{ }$ AVE \\
\hline 1. Personalidad proactiva & 3.54 & 1.19 & - & $\mathbf{. 5 0}$ & $\mathbf{. 4 5}$ & $\mathbf{. 6 0}$ & $\mathbf{. 4 8}$ & $\mathbf{. 5 8}$ & .72 & .84 \\
2. CP proorganizacionales & 2.91 & 1.22 & .58 & - & $\mathbf{. 6 5}$ & $\mathbf{. 6 4}$ & $\mathbf{. 5 8}$ & $\mathbf{. 4 0}$ & .70 & .83 \\
3. CP prosociales & 3.35 & 1.36 & .51 & .47 & - & $\mathbf{. 6 3}$ & $\mathbf{. 5 3}$ & $\mathbf{. 4 1}$ & .71 & .83 \\
4. CP proindividuales & 3.56 & 1.27 & .47 & .61 & .55 & - & $\mathbf{. 6 7}$ & $\mathbf{. 5 5}$ & .76 & .87 \\
5. Implicación laboral & 3.48 & 1.29 & .52 & .53 & .43 & .47 & - & $\mathbf{. 6 6}$ & .74 & .86 \\
6. Satisfacción laboral & 3.85 & 1.24 & .62 & .50 & .48 & .49 & .51 & - & .79 & .88 \\
\hline
\end{tabular}

Nota. Los valores bajo la diagonal corresponden a la proporción heterorrasgo-monorrasgo entre factores. Negrita: $\mathrm{p}<.01$. $\mathrm{CP}=$ comportamientos proactivos. 
constructos estudiados. Del mismo modo, el valor del AVE se ubicó por encima del mínimo recomendado y en todos los casos la proporción HTMT fue inferior a .90 (Cheung \& Chang, 2017; Henseler et al., 2015; Martínez-García \& Martínez-Caro, 2009). En conjunto, estos resultados proporcionan apoyo empírico a la hipótesis 1 , que adelantó que los comportamientos proactivos (proindividuales, prosociales y proorganizacionales), tal como son operacionalizados en la escala homónima de Belschak y Den Hartog (Salessi \& Omar, 2018a) serían un constructo empíricamente distinguible de la personalidad proactiva. En ese sentido, apoyan la validez discriminante del instrumento.

\section{Análisis de validez predictiva e incremental}

Los índices de ajuste calculados para el primer modelo fueron satisfactorios (CFI $=.86, \mathrm{TLI}=$ .89, RMSEA $=.04$, IC 95\% [.03, .05]). La personalidad proactiva realizó una contribución significativa explicando el $39 \%\left(R^{2}=.39, f^{2}=.28 ; \beta=.58, p<.001\right)$ de la varianza de la satisfacción y el $20 \%\left(R^{2}=\right.$ $\left..20, f^{2}=.16 ; \beta=.48, p<.001\right)$ de la varianza de la implicación en el trabajo.

El segundo modelo también mostró un ajuste adecuado (CFI $=.93, \mathrm{TLI}=.92$, RMSEA $=.04$, IC $95 \%[.03, .05])$. Los coeficientes de regresión estandarizados fueron significativos para los tres comportamientos proactivos, tanto en la explicación de la satisfacción laboral (comportamientos proindividuales: $\beta=.30, p<.001$; comportamientos prosociales: $\beta=.19, p<.001$; comportamientos proorganizacionales: $\beta=.12, p=.001$ ), como de la implicación en el trabajo (comportamientos proindividuales: $\beta=$ $.36, p<.001$; comportamientos prosociales: $\beta=.18, p<.001$; comportamientos proorganizacionales: $\beta$ $=.15, p<.001)$. El porcentaje de varianza explicada se elevó un $14 \%$ para la satisfacción $\left(R^{2}=.53, f^{2}=\right.$ $.35)$ y un $13 \%$ para la implicación en el trabajo $\left(R^{2}=.33, f^{2}=.25\right)$. En lo que respecta a la validez incremental, los valores de $R^{2}$ y del $f^{2}$ sugieren que el modelo que incluyó a los comportamientos proactivos alcanzó un tamaño de efecto superior.

De forma paralela, el tercer modelo estimado mostró un ajuste apropiado (CFI $=.87$, TLI $=.88$, $R M S E A=.03$, IC 95\% $[.02, .04])$. Concretamente, la personalidad proactiva explicó el $37 \%\left(R^{2}=.37, f^{2}=\right.$ $.27, \beta=.61, p<.001)$ de los comportamientos orientados al individuo; el $25 \%$ de los comportamientos orientados a la organización $\left(R^{2}=.25, f^{2}=.20, \beta=.50, p<.001\right)$ y el $20 \%$ de los comportamientos orientados a los demás $\left(R^{2}=.20, f^{2}=.16, \beta=.45, p<.001\right)$.

En conjunto, los resultados proporcionan apoyo empírico a la hipótesis 2, la cual anticipó que la personalidad proactiva explicaría una porción significativa de la varianza de la satisfacción laboral, de la implicación en el trabajo y de los comportamientos proactivos. Asimismo, los hallazgos también confirman la hipótesis 3 , desde el momento que los comportamientos proactivos realizaron un aporte significativo incremental más allá de la personalidad a la explicación de la satisfacción y de la implicación de los trabajadores.

\section{Discusión}

En los últimos años, diversos especialistas (Le et al., 2010; Morrow, 1983; Rousseau, 2007; Schwab, 1980) advierten sobre la proliferación indiscriminada de conceptos en la investigación organizacional. Del mismo modo, evidencias preliminares (Torneau \& Frese, 2013; Thomas et al., 2010) muestran que algunos de los constructos que conforman la red nomológica de la proactividad presentan cierta 
Escala Comportamientos Proactivos: evidencias de validez

redundancia. Frente a este panorama, el presente estudio se propuso contribuir a la literatura. Se analiza la validez discriminante, predictiva e incremental de los comportamientos proactivos, tal como son los operacionalizados por la escala homónima desarrollada por Belschak y Den Hartog (2010) y adaptada para el contexto argentino por Salessi y Omar (2018a).

En lo que hace a la validez discriminante, la exploración preliminar mediante el análisis ESEM indicó que un modelo de medida integrado por seis factores latentes correlacionados, con sus respectivos ítems como variables observables y los correspondientes errores de medición, no difiere significativamente de la matriz de covarianza de los datos. Todos los ítems pesaron notablemente más en los factores latentes esperados. Aun cuando las saturaciones cruzadas no fueron nulas, en ningún caso superaron el límite establecido (Marsch et al., 2014). Sumado a esto, los valores obtenidos en los índices AVE, en sus respectivas raíces cuadradas y en las proporciones HTMT mostraron que más del 50\% de sus varianzas son atribuibles a los indicadores concernientes y que, a su vez, cada constructo latente comparte más varianza con sus propios indicadores que con los demás (Cheung \& Chang, 2017; Henseler et al., 2015; Martínez-García \& Martínez-Caro, 2009). Paralelamente, el análisis de correlaciones efectuado mostró vinculaciones significativas entre todas las variables estudiadas; en ningún caso fueron próximas a 1.

Asimismo, el análisis de ecuaciones estructurales confirmó la validez predictiva e incremental de la Escala de Comportamientos Laborales Proactivos (Belschak \& Den Hartog, 2010; Salessi \& Omar, 2018a) por sobre la Escala de Personalidad Proactiva (Bateman \& Crant, 1993; Salessi \& Omar, en prensa). En este sentido, se observó que las puntuaciones en la escala de comportamientos proactivos realizan un incremento significativo a la varianza explicada, tanto de la satisfacción como de la implicación en el trabajo. En ambos casos, dicha contribución fue ligeramente superior para los comportamientos proindividuales. Tales resultados se encuentran en sintonía con hallazgos previos (Belschak \& Den Hartog, 2010; Torneau \& Frese, 2013), que ya habían iluminado su capacidad explicativa más allá de la personalidad proactiva. Asimismo, coinciden con la literatura publicada (Cangiano \& Parker, 2016; Ismaeli, et al., 2016; Mallin et al., 2014; Salessi \& Omar, 2018a; Thomas et al., 2010; Tornau \& Frese, 2013), de la cual se desprende el efecto positivo que los comportamientos proactivos ejercen sobre las actitudes laborales.

En el presente estudio, las puntuaciones en la escala de personalidad proactiva también contribuyeron en la explicación de los comportamientos proactivos, aunque tal contribución fue levemente superior para los comportamientos individuales. Estos hallazgos avalan las conclusiones de comunicaciones previas (Bergeron et al., 2013; Crant et al., 2017; Tornau \& Frese, 2013; Parker \& Collins, 2010; Spitzmuller et al., 2015), que han informado que la predisposición a controlar el ambiente influye directamente en la manifestación de acciones orientadas al cambio.

En conjunto, los resultados proporcionan apoyo empírico a la hipótesis 1 (que postulaba la validez discriminante de la escala de comportamientos proactivos); a la hipótesis 2 (que adelantaba el rol explicativo de la personalidad proactiva sobre la satisfacción laboral, la implicación en el trabajo y los comportamientos proactivos) y a la hipótesis 3 (que anticipaba la validez predictiva-incremental de los comportamientos proindividuales, prosociales y proorganizacionales en la explicación de las variables criterio). Si bien tales resultados se erigen como evidencias favorables a la validez del instrumento aquí 
analizado cabe destacar que, en línea con el debate contemporáneo, la validez es una cuestión de grado (Montero-Rojas, 2013). De acuerdo con Messick (1989) la validez no es una propiedad intrínseca de los instrumentos. Se define según el propósito de la medición, la población a la que se dirige y el contexto específico de aplicación. Así, un instrumento puede exhibir un grado aceptable de validez para un propósito específico y una población particular, pero no para otros. En otras palabras, la validez no es una cuestión de "todo o nada". No se puede decir de manera contundente que una prueba es válida, más bien exhibe un grado aceptable de validez para ciertos usos específicos y con ciertas poblaciones. Por ende, es preciso concluir que los hallazgos del presente estudio deben ser interpretados con cautela y dentro de los límites de la investigación ejecutada.

\section{Limitaciones, fortalezas e implicancias prácticas del estudio}

Entre las principales limitaciones de la investigación realizada se cita la representatividad de la muestra, puesto que, al haber sido seleccionada en forma no probabilística, impide generalizar los resultados al conjunto de la población. Sin embargo, se destaca que con el propósito de minimizar este inconveniente se procuró que la muestra en estudio incluyera empresas e instituciones de los más diferentes rubros y niveles de complejidad organizacional. Del mismo modo, el diseño transversal utilizado impide cualquier inferencia de causalidad estricta entre las variables estudiadas. Por lo tanto, sería valioso que futuras investigaciones emplearan diseños longitudinales con miras a establecer el orden causal de las relaciones aquí encontradas. Por último, dado que en el presente estudio se han utilizado instrumentos de autoinforme para evaluar las variables bajo análisis, las respuestas podrían encontrarse contaminadas tanto por la tendencia a aparecer mejor como por otros componentes subjetivos. Para superar esta limitación sería recomendable que futuros estudios incluyeran una medida de deseabilidad social como recurso de validez interna, así como información provista por otros métodos y/o informantes.

Más allá de estas limitaciones, el trabajo realizado representa una contribución genuina a la literatura organizacional. Esta investigación constituye la primera tentativa de analizar la validez discriminante, predictiva e incremental de la Escala de Comportamientos Laborales Proactivos (Belschak \& Den Hartog, 2010; Salessi \& Omar, 2018a) en relación con otras escalas de personalidad proactiva, satisfacción e implicación en el trabajo.

De los hallazgos del presente estudio, también derivan implicancias, tanto para los estudiosos del comportamiento organizacional como para gerentes y profesionales de recursos humanos. Para los primeros, establecer la validez de un instrumento conlleva una serie de beneficios prácticos. En este sentido, disponer de evidencias de validez convergente-discriminante permite suponer que la varianza de los constructos latentes medidos por un instrumento es atribuible a sus indicadores observables y que el constructo es empíricamente distinto de otros (Cheung \& Chang, 2017; Henseler et al., 2015). En una palabra, el instrumento mide lo que pretende medir (Martinez-García \& Martínez-Caro, 2009). Del mismo modo, establecer la validez predictiva e incremental posibilita estimar cuantitativamente el grado en que un constructo incrementa la capacidad explicativa y determinar si esta varianza única es significativa. En última instancia, permite verificar el grado en el que la inclusión de diversas medidas en el proceso de evaluación mejora consistentemente el poder predictivo y, en consecuencia, la toma de decisiones (Sackett et al., 2017). 
Escala Comportamientos Proactivos: evidencias de validez

Para los gerentes y los responsables de recursos humanos, los resultados iluminan la relevancia de las conductas proactivas para la satisfacción y la implicación de los trabajadores. Frente a tales evidencias, las organizaciones preocupadas por incrementar su productividad y contribuir al bienestar de sus miembros deberían concentrarse en la creación de un entorno generador de proactividad. Al respecto, la literatura científica (Belschak \& Den Hartog, 2017; Crant et al., 2017; Parker \& Bindl, 2017; Parker \& Wang, 2015) destaca el impacto de las variables ligadas a la cultura organizacional y al diseño del trabajo. En este sentido, las organizaciones, que promueven el empoderamiento, la participación de los empleados y el desarrollo de competencias, favorecen la ejecución de comportamientos proactivos en sus miembros. Del mismo modo, procesos de trabajos flexibles y puestos enriquecidos por la autonomía y la diversidad de tareas predisponen favorablemente a la proactividad; posiblemente, dada la mayor responsabilidad y el reto que tales puestos suponen. Finalmente, aquellos trabajadores que perciben que sus contribuciones son valoradas y estimuladas se muestran proclives a involucrase en acciones proactivas; principalmente, en aquellas orientadas a beneficiar a la organización.

Para concluir, se señala que la investigación contemporánea (Belschak \& Den Hartog, 2017; Grant \& Ashford, 2008; Parker \& Wang, 2015) sostiene que la variabilidad interindividual de la proactividad no se debe tanto a las diferencias en rasgos fijos y estables, sino a características maleables y abiertas al cambio. De aquíque sealícito sostenerque, enmayoromenormedida, todaslas personaspueden serproactivas en diversas circunstancias y, paralelamente, que la proactividad pueda ser desarrollada con intervenciones específicas.

\section{Referencias}

American Psychological Association (2017). Ethical principles of psychologists and code of conduct. Recuperado de: http://www.apa.org/ethics/code/

American Educational Research Association, American Psychological Association, \& National Council on Measurement in Education. (2014). Standards for educational and psychological testing. Washington, DC: American Psychological Association

Ato, M., López, J., \& Benavente, A. (2013). Un sistema de clasificación de los diseños de investigación en psicología. Anales de Psicología, 29(3), 1038-1059. doi: http://dx.doi.org/10.6018/analesps.29.3.178511

Bateman, T. S., \& Crant, J. M. (1993). The proactive component of organizational-behaviour: A measure and correlates. Journal of Organizational Behaviour, 14, 103-118. doi: https://doi.org/10.1002/job.4030140202

Belschak, F. D., \& Den Hartog, D. N. (2010). Pro-self, prosocial, and pro-organizational foci of proactive behaviour: Differential antecedents and consequences. Journal of Occupational and Organizational Psychology, 83, 475-498. doi: https://doi.org/10.1348/096317909X439208

Belschak, F., \& Den Hartog, D. (2017). Foci of proactive behaviour. In S. Parker, \& U. Bindl, (Eds.), Proactivity at work: Making things happen in organizations (pp. 169-189). New York, NY: Routledge.

Bergeron, D., Schroeder, T., \& Martinez, H. (2014). Proactive personality at work: Seeing more to do and doing more? Journal of Business \& Psychology, 29(1), 71-86. doi: http://dx.doi.org/10.1007/s10869-013-9298-5 
Borsboom, D., Mellenbergh, G. J. \& Van Heerden, J. (2004). The concept of validity. Psychological Review, 111(4), 1061-1071. doi: http://dx.doi.org/10.1037/0033-295X.111.4.1061

Cangiano, F., \& Parker, S. K. (2016). Proactivity for mental health and well-being. In S. Clarke, T. Probst, F. Guldenmund \& J. Passmore (Eds), The Wiley Blackwell Handbook of the Psychology of Occupational Safety and Workplace Health (pp. 228-250). Oxford, UK: Wiley-Blackwell. doi: https://doi. org/10.1002/9781118979013.ch11

Crant, M. Hu, J., \& Jiang, K. (2017). Proactive personality: A twenty-year review. In S. Parker, \& U. Bindl, (Eds.), Proactivity at work: Making things happen in organizations (pp. 194-225). New York, NY: Routledge

Cheung, G. \& Chang, W. (2017). Current approaches for assessing convergent and discriminant validity with SEM: Issues and solutions. Academy of Management Annual Meeting Proceedings, 2017(1), 1. doi: https://doi. org/10.5465/AMBPP.2017.12706abstract

Cohen, J. (1992). A power primer. Psychological Bulletin, 112(1), 155-159. doi: http://dx.doi.org/10.1037/00332909.112.1.155

Consejo Nacional de Investigaciones Científicas y Técnicas (CONICET, 2006). Resolución D N. ${ }^{\circ} 2857$ : CONICET: Lineamientos para el comportamiento ético en las Ciencias Sociales y Humanidades. Buenos Aires: CONICET.

Cooper-Thomas, H. D., Paterson, N., Stadler, M., \& Saks, L. (2014). The relative importance of proactive behaviours and outcomes for predicting newcomer learning, well-being, and work engagement. Journal of Vocational behaviour, 84(3), 318-331. doi: https://doi.org/10.1016/j.jvb.2014.02.007

Grant, A. M. \& Ashford, S. J. (2008). The dynamics of proactivity at work. Research in Organizational Behaviour 28, 3-34. doi: https://doi.org/10.1016/j.riob.2008.04.002

Hair, J. E., Black, W. C., Babin, B. J., Anderson, R. E., \& Tatham, R. L. (2010). Multivariate data analysis (6th ed.). Upper Saddle River, NJ: Pearson-Prentice Hall.

Harter, J. K., \& Schmidt, F. L. (2008). Conceptual versus empirical distinctions among constructs: Implications for discriminant validity. Industrial and Organizational Psychology: Perspectives on Science and Practice, 1, 36-39. http://dx.doi.org/10.1111/j.1754-9434.2007.00004.x

Henseler, J., Ringle, C. M., \& Sarstedt, M. (2015). A new criterion for assessing discriminant validity in variancebased structural equation modelling. Journal of the Academy of Marketing Science, 43(1), 115-135. doi: https://doi.org/10.1007/s11747-014-0403-8

Hunsley, J., \& Meyer, G. (2003). The incremental validity of psychological testing and assessment: conceptual, methodological and statistical issues. Psychological Assessment, 15(4), 446-455. doi: http://dx.doi. org $/ 10.1037 / 1040-3590.15 .4 .446$

Ismaeli, A., Nowalid, W., \& Bakar, R. (2016). Proactive behaviour as a mediator of the relationship between career management and career satisfaction. Journal Pengurusan, 48(1), 1-19.

Jiang, Z. (2017). Proactive personality and career adaptability: The role of thriving at work. Journal of Vocational behaviour, 98(1), 85-97. doi: https://doi.org/10.1016/j.jvb.2016.10.003 
Escala Comportamientos Proactivos: evidencias de validez

Kanungo, R. N. (1982). Measurement of job and work involvement. Journal of Applied Psychology, 67(3), 341349. doi: http://dx.doi.org/10.1037/0021-9010.67.3.341

Le, H., Schmidt, F., Harter, J., \& Lauver, K. (2010). The problem of empirical redundancy of constructs in organizational research: An empirical investigation. Organizational behaviour and Human Decision Processes 112(2), 112-125. doi: http://dx.doi.org/10.1016/j.obhdp.2010.02.003

Liao, P. (2015). The Role of self-concept in the mechanism linking proactive personality to employee work outcomes. Applied Psychology: An International Review, 64(2), 421-443. doi: https://doi.org/10.1111/ apps. 12003

Macdonald, S., \& MacIntyre, P. (1997). The generic job satisfaction scale: Scale development and its correlates. Employee Assistance Quarterly, 13(2), 1-16. doi: https://doi.org/10.1300/J022v13n02_01

Mallin, M., Ragland, C., \& Finkle, T. (2014). The proactive behaviour of youngest salespeople: Antecedents and outcomes. Journal of Marketing Channels, 21(4), 268-278. doi: https://doi.org/10.1080/104666 $\underline{\text { 9X.2014.945359 }}$

Marsh, H., Morin, A., Parker, P., \& Kaur, G. (2014). Exploratory structural equation modeling: An integration of the best features of exploratory and confirmatory factor analysis. Annual Review of Clinical Psychology, 10, 85-110. doi: https://doi.org/10.1146/annurev-clinpsy-032813-153700

Martínez-García, J. \& Martínez-Caro, L. (2009). La validez discriminante como criterio de evaluación de escalas: ¿teoría o estadística? Universitas Psychologica, 8(1), 27-36. Recuperado de: http://www.redalyc.org/ pdf/647/64712168003.pdf

Messick, S. (1989). Test validity and the ethics of assessment. American psychologist, 35(11), 1012-1027. doi: http://dx.doi.org/10.1037/0003-066X.35.11.1012

Montero-Rojas, E. (2013). Referentes conceptuales y metodológicos sobre la noción moderna de validez de instrumentos de medición: implicaciones para el caso de personas con necesidades educativas especiales. Actualidades en Psicología, 27(114), 113-128. doi: https://doi.org/10.15517/ap.v27i114.7900

Morrow, P. C. (1983). Concept redundancy in organizational research: The case of work commitment. Academy of Management Review, 8(1), 486-500. doi: https://doi.org/10.5465/amr.1983.4284606

Newton, P. E., \& Shaw, D. S. (2016). Disagreement over the best way to use the word 'validity' and options for reaching consensus. Assessment in Education: Principles, Policy and Practice, 23(2), 178-197. doi: https:// doi.org/10.1080/0969594X.2015.1037241

Parker, S., \& Bindl, U. (2017). Proactivity at work: A big picture perspective on a construct that matters. In S. K. Parker \& U. Bindl, U. (Eds.), Proactivity at work: Making things happen in organizations (pp. 1-20). New York, NY: Routledge. doi: https://doi.org/10.4324/9781315797113

Parker, S. K., \& Collins, C. (2010). Taking stock: Integrating and differentiating multiple proactive behaviours. Journal of Management, 36(3), 633-662. doi: https://doi.org/10.1177/0149206308321554

Parker, S. K. \& Wang, Y. (2015). Helping people to 'make things happen': A framework for proactivity at work. International Coaching Psychology Review, 10(1), 62-75. 
Rousseau, D. M. (2007). A sticky, leveraging, and scalable strategy for high-quality connections between organizational practice and science. Academy of Management Journal, 50(5), 1037-1042. doi: https://doi. org/10.5465/amj.2007.27155539

Sackett P., Dahlke J., Shewach O., \& Kuncel, N. (2017). Effects of predictor weighting methods on incremental validity. The Journal of Applied Psychology, 102(10), 1421-1434. doi: http://dx.doi.org/10.1037/apl0000235

Salessi, S., \& Omar, A. (2016). Satisfacción laboral genérica. Propiedades psicométricas de una escala para medirla. Revista Alternativas en Psicología, 34(1), 93-108.

Salessi, S., \& Omar, A. (2018a). Comportamientos proactivos en el trabajo: validación y análisis psicométrico de una escala. Actualidades en Psicología, 32(124), 33-49. doi: http://dx.doi.org/10.15517/ap.v32i124.30642

Salessi, S., \& Omar, A. (2018b). Implicación en el trabajo: validez y confiabilidad de la escala de Kanungo en Argentina. Revista Mexicana de Investigación Psicológica, 35(2), 179-192.

Salessi, S., \& Omar, A. (en prensa). Propiedades psicométricas de la versión argentina de la Escala de Personalidad Proactiva. Psico-USF.

Schwab, D. E. (1980). Construct validity in organizational behaviour. In B. M. Staw \& L. L. Cummings (Eds.). Research in organizational behaviour (Vol. 2, pp. 3-43). Greenwich, CT: JAI Press.

Spitzmuller, M., Sin, H., Howe, M., \& Fatimah, S. (2015). Investigating the uniqueness and usefulness of proactive personality in organizational research: A meta-analytic review. Human Performance, 28(4), 351-379. doi: https://oi.org/10.1080/08959285.2015.1021041

Tabachnick, B. G., \& Fidell, L. S (2013). Using Multivariate Statistics (6th ed.). Boston, MA: Pearson.

Thomas, J., Whitman, D., \& Viswesvaran, C. (2010) Employee proactivity in organizations: A comparative metaanalysis of emergent proactive constructs. Journal of Occupational \& Organizational Psychology, 83(2), 275-300. doi: https://doi.org/10.1348/096317910X502359

Tornau, K., \& Frese, M. (2013). Construct clean-up in proactivity research: A meta-analysis on the nomological net of work-related proactivity concepts and their incremental validities. Applied Psychology: An International Review, 62(1), 44-96. https://doi.org/10.1111/j.1464-0597.2012.00514.x

Turban, D., Moake, T., Wu, S., Cheung, Y., \& Yu, H. (2017). Linking extroversion and proactive personality to career success. Journal of Career Development, 44(1), 20-33. doi: https://doi.org/10.1177/0894845316633788

Vandenberghe, C., \& Ok, A. (2013). Career commitment, proactive personality, and work outcomes: A cross-lagged study. Career Development International, 18(7), 652-672. doi: https://doi.org/10.1108/CDI-02-2013-0013

Wang, Z., Zhang, J., Thomas, C., Yu, J., \& Spitzmueller, C. (2017). Explaining benefits of employee proactive personality: The role of engagement, team proactivity composition and perceived organizational support. Journal of Vocational behaviour, 101(1), 90-103. https://doi.org/10.1016/j.jvb.2017.04.002

Yang, K., Yan, X., Fan, J., \& Luo, Z. (2017). Leader-follower congruence in proactive personality and work engagement: A polynomial regression analysis. Personality and Individual Differences, 105(1), 43-46. doi: https://doi.org/10.1016/j.paid.2016.09.033 


\section{Sobre las autoras}

Solana Salessi es doctora en Psicología por la Universidad Nacional de La Plata, Argentina. Se desempeña como becaria posdoctoral del Consejo Nacional de Investigaciones Científicas y Técnicas (CONICET). Labora como docente de grado y posgrado en la Universidad Nacional de Rafaela y en la Universidad Católica Argentina. Dirige el Instituto de Investigaciones de Psicología de la Universidad Católica de Santa Fe. Sus intereses científicos están enfocados en el estudio de procesos psicosociales aplicados al campo de la psicología organizacional, en psicometría y en métodos cuantitativos de investigación. Autora de artículos en revistas especializadas y capítulos de libro por invitación.

Alicia Omar es doctora en Psicología por la Universidad Nacional de San Luis, Argentina. Se desempeña como investigadora científica del Consejo Nacional de Investigaciones Científicas y Técnicas (CONICET) en el Instituto de Investigaciones de la Facultad de Humanidades y Artes de la Universidad Nacional de Rosario. Es profesora invitada de universidades argentinas y extranjeras para dictar seminarios sobre metodología de la investigación y comportamiento organizacional. Sus intereses científicos están enfocados en el estudio de los procesos psicosociales aplicados a la psicología social y organizacional. Autora de libros, capítulos de libros por invitación y artículos en revistas especializadas. 